\title{
Soil-Transmitted Helminth Infection and Nutritional Status Among Urban Slum Children in Kenya
}

\author{
Parminder S. Suchdev, * Stephanie M. Davis, Monina Bartoces, Laird J. Ruth, Caitlin M. Worrell, Henry Kanyi, \\ Kennedy Odero, Ryan E. Wiegand, Sammy M. Njenga, Joel M. Montgomery, and LeAnne M. Fox \\ Division of Nutrition, Physical Activity and Obesity and Division of Parasitic Diseases and Malaria, Centers for Disease Control and Prevention, \\ Atlanta, Georgia; Department of Pediatrics, School of Medicine, Emory University, Atlanta, Georgia; Eastern and Southern Africa Centre \\ for International Parasite Control, Kenya Medical Research Institute, Nairobi, Kenya; Global Disease Detection and Emergency \\ Response, Centers for Disease Control and Prevention, Nairobi, Kenya
}

\begin{abstract}
To evaluate the nutritional impact of soil-transmitted helminth (STH) infection, we conducted a crosssectional survey of 205 pre-school (PSC) and 487 school-aged children (SAC) randomly selected from the surveillance registry of the Centers for Disease Control and Prevention of the Kibera slum in Kenya. Hemoglobin, iron deficiency (ID), vitamin A deficiency (VAD), inflammation, malaria, anthropometry, and STH ova were measured. Poisson regression models evaluated associations between STH and malnutrition outcomes and controlled for confounders. Approximately $40 \%$ of PSC and SAC had STH infection, primarily Ascaris and Trichuris; $2.9 \%$ of PSC and $1.1 \%$ of SAC had high-intensity infection. Malnutrition prevalence among PSC and SAC was anemia $(38.3 \%$ and $14.0 \%$, respectively), ID $(23.0 \%$ and $5.0 \%$, respectively), VAD (16.9\% and $4.5 \%$, respectively), and stunting (29.7\% and $16.9 \%$, respectively). In multivariate analysis, STH in PSC was associated with VAD (prevalence ratio [PR] $=2.2$, $95 \%$ confidence interval $=1.1-4.6)$ and ID $(\mathrm{PR}=3.3,95 \%$ confidence interval $=1.6-6.6)$ but not anemia or stunting. No associations were significant in SAC. Integrated deworming and micronutrient supplementation strategies should be evaluated in this population.
\end{abstract}

\section{INTRODUCTION}

Soil-transmitted helminths (STHs) are parasitic intestinal worms that infect more than one-half of the world's population. ${ }^{1}$ The most common species are Ascaris lumbricoides, Trichuris trichiura, and hookworm, which chronically infect children and are associated with significant physical, cognitive, and economic deficits. ${ }^{2}$ Infection with STH causes more morbidity than death, contributing to as many as 405,000 deaths and 37 million disability-adjusted life years (DALYs) annually. ${ }^{3}$

World Health Organization (WHO) guidelines for STH control generally focus on routine mass drug administration for school-aged children, aiming primarily to reduce the prevalence of high-intensity infection. ${ }^{4}$ Although school-based treatment is currently a priority, pre-school children also experience substantial morbidity and may benefit from deworming., 5 Another challenge for STH control is how to target interventions given the geographical heterogeneity of infection in countries, communities, and individuals, in which different species of STH often co-occur. ${ }^{7}$ Guidance is also needed for urban slums, which house nearly one-third of the world's population and are less studied for STH interventions. ${ }^{8}$

The proven burden of chronic STH infection is primarily related to malnutrition and impaired growth. ${ }^{1,9}$ For example, there are proven effects of STH infection on iron status, vitamin A status, anemia, and growth that are mediated through various mechanisms, including malabsorption, inflammation, and reduced food intake from reduced appetite. ${ }^{1,5,9}$ However, few studies have explored the association of STH and malnutrition while accounting for multiple covariates and severity of STH infection. ${ }^{9}$ Furthermore, the data on the burden of STH in the urban slum populations are limited. We, there-

*Address correspondence to Parminder S. Suchdev, Nutrition Branch, Centers for Disease Control and Prevention, 4770 Buford Highway NE, MS-F77, Atlanta, GA 30341. Email: psuchdev@cdc.gov fore, aimed to evaluate the association of STH infection with anemia, iron deficiency, vitamin A deficiency, and growth among populations of pre-school- and school-aged children living in an urban slum in Kenya. We also aimed to evaluate the association of STH and inflammation to explore potential mechanisms by which STH infection may cause malnutrition.

\section{METHODS}

Study population and sample. The study was conducted in Kibera in southern Nairobi, Kenya, which is one of the largest contiguous urban slums in Africa. ${ }^{10}$ The Centers for Disease Control and Prevention's International Emerging Infections Program (IEIP), in collaboration with the Kenya Medical Research Institute (KEMRI), has conducted communitybased morbidity surveillance in Kibera since 2005. ${ }^{11}$ IEIP performs active household surveillance for major infectious disease syndromes for approximately 28,000 participants living in 2 of Kibera's 13 villages. Field workers make home visits every 2 weeks to ask about recent illnesses; they also maintain an up-to-date registry by enrolling new arrivals and updating records to reflect outmigration. ${ }^{10,11}$ Kibera is characterized by high population density, semipermanent housing, and lack of official city water or sewage services. Participants can access free healthcare at a centrally located clinic staffed by study-supported trained personnel and other clinics run by non-governmental organizations and government institutions bordering the slum.

Among households in the IEIP participant registry, approximately $25 \%$ of households were designated as potential sources for enrollment of pre-school children (PSC; ages 659 months), and the other $75 \%$ of households were designated as potential sources for enrollment of school-aged children (SAC; ages 5-14 years); this designation ensured that PSC and SAC were not from the same household. Households were then selected with probability proportional to size from each target group, and one child was randomly chosen from 
each selected household. Sampling weights were calculated to account for non-response and post-stratified to the IEIP cluster size populations. Sample size was capped by the laboratory capacity for daily processing of fresh stools for STH. At $80 \%$ power and accounting for $20 \%$ non-response, the achievable target sample size of 293 PSC and 899 SAC was powered to detect an odds ratio of 2.0 for STH infection associated with having an infected sibling.

Written informed consent was obtained from all participating households. Subjects with anemia, positive malaria test, wasting, or STH infection were referred for free care at the nearby IEIP-run clinic. Institutional review boards of KEMRI and the Centers for Disease Control and Prevention (CDC) approved this study.

Data collection. Fieldwork took place from April to June of 2012. Trained fieldworkers administered a mobile devicebased questionnaire to obtain demographic and socioeconomic data and child breastfeeding, deworming, and nutrition supplementation history. Anthropometric measurements of height and length were taken using a wooden measuring board accurate to $0.1 \mathrm{~cm}$ (Irwin Shorr Productions, Olney, MD). Weight was measured to the nearest $0.1 \mathrm{~kg}$ using a digital scale (Seca Corp, Hanover, MD). Trained fieldworkers completed the measurements using standard techniques. Capillary blood samples were collected for hemoglobin $(\mathrm{Hb})$ measurements, malaria testing, and analysis of micronutrient and inflammatory status.

A goal of three stool samples was collected from selected children. Stools were collected over 3 consecutive days when possible, accepted only if produced after midnight the previous night, and maintained in cool boxes before delivery to the laboratory for analysis before 14:00 hours each day. Stool samples were processed at KEMRI using the Kato-Katz technique. Two slides were prepared per sample; approximately $7 \%$ of all slides were reread for quality control.

Details of the nutrition laboratory analysis are described elsewhere. ${ }^{12}$ In brief, $\mathrm{Hb}$ was determined using a HemoCue

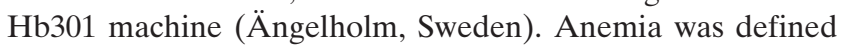
by age and corrected for altitude according to WHO guidelines ${ }^{13}: \mathrm{Hb}<11.0 \mathrm{~g} / \mathrm{dL}$ in children $6-59$ months of age, $\mathrm{Hb}<$ $11.5 \mathrm{~g} / \mathrm{dL}$ in children $5-11$ years of age, and $\mathrm{Hb}<12 \mathrm{~g} / \mathrm{dL}$ in children 12-14 years of age; $0.6 \mathrm{~g} / \mathrm{dL}$ was added to cutoffs to account for an altitude of $1,800 \mathrm{~m}$. A rapid diagnostic test kit (SD BIOLINE Malaria Ag P.f/Pan, Hagal-dong, Korea) was used to test for malaria. Blood samples were centrifuged, and frozen plasma samples were transported to VitA-Iron Tech (Willstaett, Germany), where levels of ferritin, retinol binding protein (RBP), C-reactive protein (CRP), and $\alpha$-1-acid glycoprotein (AGP) were measured by sandwich enzyme-linked immunosorbent assay (ELISA). ${ }^{14}$ The following thresholds were used to define abnormal values for these biochemical indicators: ferritin $<12 \mu \mathrm{g} / \mathrm{L}$ in PSC and ferritin $<15 \mu \mathrm{g} / \mathrm{L}$ in $\mathrm{SAC}^{15} ; \mathrm{RBP}<0.7 \mu \mathrm{mol} / \mathrm{L} ; \mathrm{CRP}>5 \mathrm{mg} / \mathrm{L} ; \mathrm{AGP}>1 \mathrm{~g} / \mathrm{L} .{ }^{16}$ Because biomarkers of nutrition are influenced by inflammation, iron deficiency, vitamin A deficiency, and anemia were defined using a correction factor approach to adjust ferritin, $\mathrm{RBP}$, and $\mathrm{Hb}$ values for the presence of inflammation, which was described previously. ${ }^{16,17}$ Because the prevalence of inflammation varied by age, separate correction factors were calculated for PSC and SAC. Among PSC, correction factors for ferritin, $\mathrm{RBP}$, and $\mathrm{Hb}$ were as follows: 0.87, 1.0, and 0.91 for early inflammation (elevated CRP and normal AGP);
$0.36,1.51$, and 1.08 for early convalescent inflammation (elevated CRP and AGP); 0.78, 1.1, and 1.0 for late convalescent inflammation (elevated AGP and normal CRP). Among $\mathrm{SAC}$, correction factors for ferritin, $\mathrm{RBP}$, and $\mathrm{Hb}$ were as follows: $0.70,1.36$, and 1.01 for early inflammation; 0.66, 1.36, and 1.09 for early convalescent inflammation; $0.99,1.21$, and 1.08 for late convalescent inflammation.

Statistical analysis. We used the WHO Child Growth Standards (WHO Anthro, Geneva, Switzerland) to calculate $z$ scores and categorized underweight as a weight-for-age $z$-score of $<-2$, stunting as a height/length-for-age $z$-score of $<-2$, and wasting as a body mass index (BMI)-for-age $z$-score of $<-2$ to allow for comparisons between PSC and SAC. We used the weekly expenditure on food by the household to classify respondents by socioeconomic status. Children were classified as having received or not received vitamin A supplementation in the past 1 year based on written documentation when available or otherwise, respondent recollection.

Statistical analyses were performed using $\mathrm{R}$ version 2.15 .2 (R Core Team, 2012). The survey package was used to incorporate survey weights. Comparisons between PSC and SAC were performed with $\chi^{2}$ test. Means and standard deviations (SDs) were computed for continuous variables, and $t$ test was used to compare means. Multivariable analyses used Poisson regression to provide prevalence ratios (PR). ${ }^{18}$ All analyses were run separately for PSC and SAC.

Five nutritional outcomes were investigated: anemia, vitamin A deficiency (VAD), iron deficiency (ID), iron deficiency anemia (IDA), and stunting. Inflammation was also investigated as an outcome (e.g., elevated CRP and/or elevated AGP). These outcomes were dichotomized according to previously described cutoffs. Several alternative measures of the primary exposure (STH infection) were used: presence/ absence of any STH infection; presence/absence of speciesspecific infection with Ascaris or Trichuris; presence/absence of coinfection with both Ascaris and Trichuris; and intensity of infection (none, light, medium/moderate, or high according to standard egg per 1 gram-based WHO cutoffs ${ }^{19}$ using the highest-intensity species found in the case of coinfection, with eggs per 1 gram determined as an average among all the subjects'stools). Demographic and clinical confounders were determined independently for each outcome a priori based on knowledge from published literature that they were potential predictors of the various nutritional outcomes. Demographic factors considered were age, sex, maternal education, ethnicity, socioeconomic status, and breastfeeding. Clinical factors included were malaria, stunting, wasting, ID, and VAD. To test the robustness of the model, we also performed a sensitivity analysis for the VAD outcome, which included history of recent vitamin A supplementation.

Unadjusted prevalence ratios and 95\% confidence intervals were computed for association between a specific outcome and primary exposure. Age, sex, and socioeconomic status were forced in the models as potential confounders. When the primary exposure was Ascaris infection, presence of Trichuris infection was also forced in the models. The decision to include the other demographic variables and clinical variables in the models was based on at least a $10 \%$ change in the prevalence ratio of the primary exposure. Variables that were originally continuous were treated as both continuous and dichotomized variables in separate models. The results of these models were compared to assess residual confounding and 
whether associations were linear. The best models selected were those models with confidence intervals that were more precise.

\section{RESULTS}

In total, 1,192 children were targeted as potential study participants, and 844 children were enrolled. The primary reasons for non-enrollment were either household members were absent $(N=216)$ or the individuals refused to participate $(N=132)$. An additional 132 children were excluded because of missing laboratory or stool data, and 20 children withdrew, which led to a total of 692 children (205 PSC and 487 SAC) in the final analysis (net response rate of $58 \%$ ). Age, sex, and other demographics were similar among enrolled and nonenrolled children (data not shown).

The demographic and health characteristics of enrolled PSC and SAC are shown in Table 1. There were no significant differences between the two groups in most demographic characteristics. Most children were of Luo ethnicity, and over one-half were, by self-report, dewormed in the last year. Mean weekly per capita expenditure on food was USD $\$ 5.40$ in PSC households compared with USD \$4.90 in SAC households $(P=0.03)$. Approximately $40 \%$ of PSC and SAC had any STH infection, primarily consisting of Ascaris and Trichuris species. There was only one hookworm infection. The overall prevalence of high-intensity STH infection was low, with no greater high-intensity infection with Ascaris in PSC compared with SAC (2.9\% versus $1.1 \%, P=0.09)$. There were no cases of high-intensity infection with Trichuris, and of the Trichuris infections, 97\% were light intensity infections (data not shown).

For nutritional status, compared with SAC, PSC had a significantly higher prevalence of anemia, ID, VAD, inflammation, and stunting (Table 1). Although the prevalence of wasting was overall low $(6.8 \%)$, the prevalence was higher in SAC compared with PSC $(P<0.0001)$. The prevalence of any inflammation (either elevated CRP or AGP) was higher in PSC at $48.9 \%$ (compared with $15.8 \%$ in SAC; $P<0.0001$ ).

TABLE 1

Demographic and health characteristics of enrolled PSC and SAC in the Kibera slum, Kenya, 2012

\begin{tabular}{|c|c|c|c|c|c|}
\hline \multirow[b]{2}{*}{ Characteristics } & \multicolumn{2}{|c|}{$\operatorname{PSC}(N=205)$} & \multicolumn{2}{|c|}{$\operatorname{SAC}(N=487)$} & \multirow[b]{2}{*}{$P$ value } \\
\hline & Number* & Percent or mean $\dagger$ & Number & Percent or mean $\dagger$ & \\
\hline Age, years (mean) & 205 & $3.2 \pm 0.08$ & 487 & $11.4 \pm 0.13$ & $<0.001$ \\
\hline Boys (\%) & 98 & 47.4 & 228 & 46.8 & 0.89 \\
\hline Weekly per capita food spending per household, USD (mean) & 205 & $5.4 \pm 0.21$ & 487 & $4.9 \pm 0.12$ & 0.03 \\
\hline Less than complete maternal primary school education (\%) & 52 & 25.3 & 138 & 28.5 & 0.38 \\
\hline \multicolumn{6}{|l|}{ Ethnicity (\%) } \\
\hline Luo & 137 & 67.3 & 345 & 71.6 & 0.52 \\
\hline Luhya & 42 & 21.0 & 84 & 17.0 & 0.52 \\
\hline Kisii & 14 & 6.1 & 26 & 4.9 & 0.52 \\
\hline Other & 12 & 5.6 & 32 & 6.5 & 0.52 \\
\hline Currently breastfeeding (\%) & 38 & 19.9 & - & - & $\mathrm{NC}$ \\
\hline Dewormed in the past 1 year (\%) & 122 & 59.5 & 268 & 54.6 & 0.24 \\
\hline Positive malaria (\%) & 23 & 10.8 & 22 & 4.6 & 0.003 \\
\hline \multicolumn{6}{|l|}{ STH indicators (exposures) } \\
\hline Presence of any STH infection (\%) & 82 & 40.5 & 193 & 40.7 & 0.97 \\
\hline Ascaris infection & 50 & 24.1 & 109 & 22.7 & 0.70 \\
\hline Trichuris infection & 47 & 24.0 & 135 & 28.8 & 0.21 \\
\hline Hookworm infection & 0 & 0.0 & 1 & 0.2 & $\mathrm{NC}$ \\
\hline \multicolumn{6}{|l|}{ Intensity of Ascaris infection (\%) } \\
\hline None & 155 & 75.9 & 378 & 77.3 & 0.09 \\
\hline Light & 11 & 5.4 & 44 & 9.2 & 0.09 \\
\hline Moderate & 33 & 15.7 & 60 & 12.4 & 0.09 \\
\hline High & 6 & 2.9 & 5 & 1.1 & 0.09 \\
\hline Presence of Ascaris/Trichuris coinfection (\%) & 15 & 7.5 & 51 & 10.8 & 0.20 \\
\hline \multicolumn{6}{|l|}{ Malnutrition indicators (outcomes) } \\
\hline Anemia§ (\%) & 78 & 38.3 & 68 & 14.0 & $<0.0001$ \\
\hline Anemia (corrected for inflammation; \%) $\mathbb{I}$ & 72 & 35.3 & 50 & 10.2 & $<0.0001$ \\
\hline IDA $(\%)$ & 22 & 10.7 & 17 & 3.5 & 0.0002 \\
\hline Elevated AGP (>1 g/L; \%) & 97 & 48.3 & 66 & 13.6 & $<0.0001$ \\
\hline Elevated CRP (> 5 mg/L; \%) & 39 & 20.6 & 34 & 7.2 & $<0.0001$ \\
\hline Any inflammation (elevated CRP or AGP; \%) & 98 & 48.9 & 76 & 15.8 & $<0.0001$ \\
\hline Low ferritin $(<12$ or $<15 \mu \mathrm{g} / \mathrm{L} ; \%)$ & 34 & 16.6 & 24 & 4.8 & $<0.0001$ \\
\hline ID (exclusion method; \%) $\|$ & 33 & 20.2 & 19 & 4.0 & $<0.0001$ \\
\hline ID (corrected for inflammation; \%) $\mathbb{T}$ & 46 & 23.0 & 25 & 5.0 & $<0.0001$ \\
\hline Low RBP $(<0.7 \mu \mathrm{g} / \mathrm{L} ; \%)$ & 53 & 26.8 & 45 & 9.2 & $<0.0001$ \\
\hline VAD (exclusion method, \%) $\|$ & 26 & 16.8 & 26 & 5.6 & $<0.0001$ \\
\hline VAD (corrected for inflammation; \%) II & 34 & 16.9 & 23 & 4.5 & $<0.0001$ \\
\hline Stunted (height-for-age $z$-score $<-2 ; \%$ ) & 59 & 29.7 & 82 & 16.9 & 0.0002 \\
\hline Underweight (weight-for-age $z$-score $<-2 ; \%$ ) & 16 & 7.8 & 17 & 11.4 & 0.26 \\
\hline Wasted (BMI-for-age $z$-score $<-2 ; \%$ ) & 3 & 1.5 & 44 & 9.0 & $<0.0001$ \\
\hline \multicolumn{6}{|c|}{$\begin{array}{l}\mathrm{NC}=\text { not calculated. } \\
* \text { Unweighted frequencies. } \\
\dagger \text { Values are percent or mean } \pm \mathrm{SD} \text { weighted for complex survey design. } \\
\$ P \text { values were computed from } \chi^{2} \text { tests for differences in proportions and } t \text { tests for differences in means. } \\
\$ \text { Corrected for altitude: } \mathrm{Hb}<11.6 \mathrm{~g} / \mathrm{dL} \text { for } 6-59 \text { months; } \mathrm{Hb}<12.1 \text { for } 5-11 \text { years; } \mathrm{Hb}<12.6 \text { for } 12-14 \text { years. } \\
\text { 【I Hb, ferritin, and RBP values were adjusted for the presence of inflammation using correction factors. } \\
\| \text { Excluding children with inflammation }(\mathrm{CRP}>5 \mathrm{mg} / \mathrm{L} \text { or } \mathrm{AGP}>1 \mathrm{~g} / \mathrm{L}) \text {. }\end{array}$} \\
\hline
\end{tabular}


TABLE 2

Bivariate analysis of four measures of STH infection and several nutritional status outcomes $\dagger$ among PSC in the Kibera slum, Kenya, 2012

\begin{tabular}{|c|c|c|c|c|c|c|}
\hline STH infection & Anemia* $n(\%)$ & $\mathrm{ID} \ddagger n(\%)$ & VAD $\$ n(\%)$ & $\operatorname{IDA} n(\%)$ & Any inflammation $\llbracket n(\%)$ & Stunting $\| n(\%$ \\
\hline \multicolumn{7}{|l|}{ Any STH infection } \\
\hline Yes & $31(37.9)$ & $21(24.9)$ & $19(23.7)$ & $9(10.7)$ & $34(41.5)$ & $27(32.8)$ \\
\hline No & $41(33.6)$ & $25(21.6)$ & 15 (12.3) & $13(10.8)$ & $64(53.9)$ & $32(27.5)$ \\
\hline$P$ value** & 0.53 & 0.59 & 0.04 & 0.98 & 0.09 & 0.43 \\
\hline \multicolumn{7}{|l|}{ Ascaris infection } \\
\hline Yes & $18(35.6)$ & 17 (33.5) & $13(27.0)$ & $7(14.0)$ & $19(38.0)$ & $18(35.2)$ \\
\hline No & $54(35.2)$ & 29 (19.6) & 21 (13.7) & $15(9.7)$ & $79(52.4)$ & $41(27.9)$ \\
\hline$P$ value** & 0.97 & 0.048 & 0.03 & 0.40 & 0.08 & 0.34 \\
\hline \multicolumn{7}{|l|}{ Trichuris infection } \\
\hline Yes & $18(38.0)$ & $13(26.1)$ & $11(23.5)$ & 7 (13.6) & $23(48.7)$ & $13(28.5)$ \\
\hline No & $54(34.5)$ & $33(22.0)$ & $23(14.8)$ & $15(9.8)$ & $75(49.0)$ & $46(30.0)$ \\
\hline$P$ value** & 0.67 & 0.56 & 0.17 & 0.46 & 0.97 & 0.85 \\
\hline \multicolumn{7}{|c|}{ Ascaris/Trichuris coinfection } \\
\hline Yes & $5(30.6)$ & $9(56.3)$ & $5(33.9)$ & $5(30.6)$ & $8(53.2)$ & $4(26.8)$ \\
\hline No & $67(35.7)$ & 37 (20.2) & $29(15.5)$ & $17(9.1)$ & $90(48.6)$ & $55(29.9)$ \\
\hline$P$ value** & 0.69 & 0.002 & 0.07 & 0.01 & 0.74 & 0.80 \\
\hline
\end{tabular}

* Corrected for altitude $(\mathrm{Hb}<11.6 \mathrm{~g} / \mathrm{dL}$ adjusted for inflammation using correction factors).

$\dagger$ Percentages are weighted for complex survey design.

$\doteqdot$ Ferritin $<12 \mu \mathrm{g} / \mathrm{L}$ adjusted for inflammation using correction factors.

$\S \mathrm{RBP}<0.7 \mu \mathrm{g} / \mathrm{L}$ adjusted for inflammation using correction factors.

II Elevated AGP $(>1 \mathrm{~g} / \mathrm{L})$ or $\mathrm{CRP}(>5 \mathrm{mg} / \mathrm{L})$.

$\|$ Height-for-age $z$-score $<-2$.

$* * P$ value computed from $\chi^{2}$ test for differences in proportions.

When correcting for the presence of inflammation using the correction factor approach, prevalences of anemia and VAD were lower compared with uncorrected values, and the prevalence of ID was higher compared with uncorrected values.

The bivariate associations between nutrient outcomes and four different measures of STH infection among PSC are shown in Table 2. There were no differences in the prevalence of anemia or IDA between those children with and without any STH infection or species-specific infection with Ascaris or Trichuris. However, the prevalences of ID and IDA were significantly higher among those children coinfected with both Ascaris and Trichuris compared with those children not coinfected. Compared with those children not infected, there was a higher prevalence of ID among those children infected with Ascaris $(P=0.048)$ and a higher prevalence of VAD among those children infected with Ascaris or any STH infection $(P=0.03$ or $P=0.04$, respectively). There was a lower prevalence of any inflammation among those children with any STH infection $(P=0.09)$ and those children with Ascaris infection $(P=0.08)$, although these findings were not statistically significant in the analysis. Also, when we compared the stages of inflammation, there were no significant associations between STH infection and either elevated CRP or elevated AGP (data not shown). Among SAC, there were no significant bivariate associations between STH infection and any of the measured nutrition outcomes, except a higher prevalence of VAD among those children infected with Ascaris $(8.0 \%$ versus $3.5 \%, P=0.0496$ ).

Results of multivariate Poisson regression analyses assessing the association of STH infection and ID, VAD, and IDA among PSC are shown in Table 3. Both any STH infection and Ascaris infection were significantly associated with VAD $(\mathrm{PR}=2.2,95 \%$ confidence interval $[95 \% \mathrm{CI}]=1.1-4.6$; $\mathrm{PR}=2.2,95 \% \mathrm{CI}=1.04-4.6$, respectively). This relationship was unchanged in the sensitivity analysis, which corrected for recent vitamin A supplementation. There was some indication of a dose-response relationship, with children with moderate-intensity Ascaris infection more likely to have VAD than those children without Ascaris infection ( $\mathrm{PR}=2.4,95 \%$ $\mathrm{CI}=1.01-5.6)$. When we added reported history of vitamin A supplementation to the models, the association between any STH, Ascaris, or infection intensity and VAD did not change

TABLE 3

Multivariate analysis assessing the association of STH infection and nutritional outcomes among PSC in the Kibera slum, Kenya, 2012

\begin{tabular}{|c|c|c|c|c|c|}
\hline Primary exposure & Outcomes & Unadjusted prevalence ratio & $95 \%$ CI & Adjusted prevalence ratio & $95 \% \mathrm{CI}$ \\
\hline Any STH infection & VAD* & 1.92 & $1.03-3.58$ & $2.23 \dagger$ & $1.11-4.58$ \\
\hline Ascaris infection & VAD* & 1.97 & $1.06-3.65$ & $2.20 \ddagger$ & $1.04-4.57$ \\
\hline \multicolumn{6}{|l|}{ Intensity of Ascaris infection } \\
\hline None & VAD* & Reference & - & Reference & - \\
\hline Light & VAD* & 1.52 & $0.53-4.36$ & $2.36 \S$ & $0.57-7.12$ \\
\hline Moderate & VAD* & 2.04 & $1.05-3.94$ & 2.43 & $1.01-5.58$ \\
\hline High & VAD* & 1.00 & $0.16-6.14$ & 1.09 & $0.07-5.30$ \\
\hline Ascaris/Trichuris coinfection & IDq & 2.78 & $1.61-4.79$ & $3.28 \|$ & $1.54-6.60$ \\
\hline Ascaris/Trichuris coinfection & IDA & 3.36 & $1.39-8.16$ & $3.10 * *$ & $1.15-7.65$ \\
\hline
\end{tabular}

$* \mathrm{RBP}<0.7 \mu \mathrm{g} / \mathrm{L}$ adjusted for the presence of inflammation using correction factors.

$\dagger$ Adjusted for sex, age, socioeconomic status, ID, and stunting.

$\doteqdot$ Adjusted for Trichuris, sex, age, socioeconomic status, ID, and stunting.

$\S$ Adjusted for Trichuris, sex, age, socioeconomic status, ID, and stunting.

If Ferritin $<12 \mu \mathrm{g} / \mathrm{L}$ adjusted for the presence of inflammation using correction factors.

$\|$ Adjusted for sex, age, socioeconomic status, VAD, stunting, malaria, and wasting.

** Adjusted for sex, age, socioeconomic status, VAD, AGP, stunting, malaria, and wasting. 
considerably (data not shown). Coinfection with Ascaris and Trichuris was significantly associated with both ID and IDA $(\mathrm{PR}=3.3,95 \% \mathrm{CI}=1.5-6.6 ; \mathrm{PR}=3.1,95 \% \mathrm{CI}=1.2-7.7$, respectively). There was no significant association of STH infection in PSC and anemia, stunting, or inflammation.

Among SAC, multivariate regression analysis showed no significant association between any STH and Ascaris/Trichuris coinfection and any of the nutritional outcomes (data not shown). There was, however, a significant positive association for anemia comparing high-intensity infection with Ascaris with no infection $(\mathrm{PR}=5.3,95 \% \mathrm{CI}=1.2-16.5)$. The presence of any STH was negatively associated with elevated AGP $(\mathrm{PR}=0.55,95 \% \mathrm{CI}=0.33-0.89)$.

\section{DISCUSSION}

In this urban slum environment, we report a high prevalence of Ascaris and Trichuris infections among both PSC and SAC, despite exposure to frequent informal deworming. To our knowledge, this study was one of the first studies to describe the association of STH and malnutrition in both PSC and SAC and account for multiple known risk factors, including inflammation and malaria, as well as severity of STH infection. We found that STH infection in PSC, even at low to moderate intensity, was associated with important micronutrient deficiencies, namely VAD and ID. STH infection in SAC, however, was not associated with measures of poor nutritional status.

The $40.7 \%$ prevalence of STH infection in SAC that we reported in Kibera was higher than the $12.9 \%$ prevalence reported among primary school children in surrounding Nairobi $^{20}$ as well as the $16.2 \%$ prevalence reported among children ages 10-18 years living in urban slum settings in western Kenya. ${ }^{21}$ These findings suggest the need to consider urban slums as separate ecological zones from their parent cities for planning deworming interventions.

The association that we observed between STH infection and ID and VAD is not surprising, because mechanisms to explain the adverse effects of helminth infection on nutrition have been proposed and include decreased appetite and nutrient intake, impaired absorption, and increased nutrient loss or altered metabolism. ${ }^{9}$ In addition, Ascaris worms express a range of retinol binding proteins, retinol dehydrogenases, and retinoic acid receptors, which use retinol for growth and development, ${ }^{22}$ although a study in Sri Lanka among adolescent girls found that Trichuris infection but not Ascaris infection was associated with lower serum vitamin A concentration compared with those girls not infected. ${ }^{23}$ WHO and United Nations Children's Fund (UNICEF) have recommended that deworming be included in vitamin A supplementation programs, ${ }^{24}$ and combined vitamin A supplementation and deworming have been shown to reduce Ascaris reinfection rates among PSC in Panama. ${ }^{25}$ Although our results support an integrated approach, additional research is needed to elucidate the timing and duration of combined interventions with vitamin $\mathrm{A}$ and deworming.

We did not find an association between STH infection and anemia, which may be because of the low prevalence of highintensity infections and hookworm infection as well as other known risk factors for anemia that were prevalent in this population (e.g., vitamin deficiencies, malaria, inflammation, and inherited blood disorders). ${ }^{26}$ Studies in Zanzibar, simi- larly, found no association between helminth infection early in life and anemia or malnutrition, which is hypothesized to be secondary to increased environmental exploration and thus, increased exposure to STH by better-nourished children. ${ }^{27}$ However, in a 12-month deworming trial of children 6-71 months of age, mebendazole improved anemia only in children $<24$ months of age, suggesting that young children may still benefit from deworming, because they are at higher risk of anemia, although at lower intensity of helminth infection. ${ }^{5}$

We found no evidence of helminth-induced inflammation and in fact, a negative association between any STH infection and AGP among SAC. Helminth infections stimulate inflammation during the initial acute phase of the primary infection or during reinfection, whereas stable, low-intensity infections may not be associated with elevated levels of acute-phase proteins. $^{28}$ Furthermore, helminths induce a Th2 cytokine response, characterized by the production of the cytokines interleukin-4 (IL-4), IL-5, IL-9, and IL-13, the antibodies immunoglobulin G1 (IgG1) and IgE, and eosinophilia, which have an anti-inflammatory effect on the host and may explain the negative association that we observed of STH with chronic inflammation. $^{29}$

Although the WHO currently recommends routine mass drug administration, there is mixed evidence on the benefits of routine deworming. For example, a recent Cochrane review of 41 trials showed that, although treatment of infected children can improve growth, routine deworming alone has no effect on growth, cognitive ability, or school attendance. ${ }^{30}$ Furthermore, the recently published de-worming and vitamin A (DEVTA) study, which dewormed 1 million PSC every 6 months in northern India for 5 years, showed no impact on hemoglobin, weight, or mortality. ${ }^{31}$ These studies have led some to question the value of deworming interventions ${ }^{32}$; however, these trials did not specifically evaluate the effects of deworming on micronutrient deficiencies, such as iron or vitamin A, which according to our study's findings, may be prevalent even in populations with light to moderate STH infection. Our results from a lower-intensity STH infection setting also challenge the current emphasis on driving down the prevalence of high-intensity STH infection, which is presumed to be the major cause of morbidity. ${ }^{33}$

This study had several limitations. First, the study was conducted in two villages of the Kibera urban slum, and therefore, the findings may not be representative of the rest of Kibera or other urban slums. Second, this study was a cross-sectional study, and therefore, causality cannot be determined. The study was also underpowered to adequately assess the associations of STH infection intensity and nutrition outcomes as well as the effects of hookworm infection, which were uncommon, likely because of low evening temperatures and the urban environment. However, the statistical approach was robust, because multiple covariates were assessed, continuous variables were used to reduce residual confounding, and prevalence ratios were used as a conservative and interpretable measure of association. Finally, the low response rate was not unexpected given the challenge of reaching households in this highly mobile urban slum setting and collecting adequate and timely stools. Although the response rate was low, analyzed and non-analyzed children were similar on assessable demographic characteristics.

In conclusion, PSC are an important target group for deworming interventions, because they have a similar STH 
burden to SAC, and even low- to moderate-intensity STH infection in this group is associated with significant micronutrient deficiencies. PSC may be reached through integrated programs, such as vitamin A campaigns, vaccination programs, and Child Health Days. ${ }^{24}$ Furthermore, in high populationdensity urban slums, the feasibility of reaching young children through community-based distribution is likely to be higher than in rural areas. This setting provides an opportunity to evaluate integrated deworming and micronutrient supplementation strategies in young children.

Received September 28, 2013. Accepted for publication November 14, 2013.

Published online December 16, 2013.

Acknowledgments: This paper is published with the permission of the Director of Kenya Medical Research Institute. The authors gratefully acknowledge the contributions of Julius Andove, Fredy Munoz, the Eastern and Southern Africa Centre of International Parasite Control (ESACIPAC) laboratory staff, the Centers for Disease Control and Prevention-Kenya Medical Research Institute community interviewers, and the residents of Soweto and Gatwekira, without whom this work would not have been possible.

Financial support: Funding for this study was obtained from U.S. Agency for International Development (USAID) through an interagency agreement with the US Centers for Disease Control and Prevention.

Disclaimer: The findings and conclusions in this report are the findings and conclusions of the authors and do not necessarily represent the official position of the Centers for Disease Control and Prevention.

Authors' addresses: Parminder S. Suchdev and Laird J. Ruth, Nutrition Branch, Centers for Disease Control and Prevention, Atlanta, GA, E-mails: psuchdev@cdc.gov and 1ruth@cdc.gov. Stephanie M. Davis, Monina Bartoces, Caitlin M. Worrell, Ryan E. Wiegand, and LeAnne M. Fox, Parasitic Diseases Branch, Centers for Disease Control and Prevention, Atlanta, GA, E-mails: vic6@cdc.gov, jze8@ cdc.gov, cworrell@cdc.gov, fwk2@cdc.gov, and lff4@cdc.gov. Henry Kanyi, Kennedy Odero, and Sammy M. Njenga, Eastern and Southern Africa Centre for International Parasite Control, Kenya Medical Research Institute, Nairobi, Kenya, E-mails: hkanyi@kemri.org, koderos80@gmail.com, and SNjenga@kemri.org. Joel M. Montgomery, Global Disease Detection and Emergency Response, Centers for Disease Control and Prevention, Nairobi, Kenya, E-mail: JMontgomery@ ke.cdc.gov

\section{REFERENCES}

1. Hall A, Hewitt G, Tuffrey V, de Silva N, 2008. A review and meta-analysis of the impact of intestinal worms on child growth and nutrition. Matern Child Nutr 4 (Suppl 1): 118-236.

2. Bethony J, Brooker S, Albonico M, Geiger SM, Loukas A, Diemert D, Hotez PJ, 2006. Soil-transmitted helminth infections: ascariasis, trichuriasis, and hookworm. Lancet 367: $1521-1532$.

3. Hotez PJ, Molyneux DH, Fenwick A, Ottesen E, Ehrlich Sachs S, Sachs JD, 2006. Incorporating a rapid-impact package for neglected tropical diseases with programs for HIV/AIDS, tuberculosis, and malaria. PLoS Med 3: e102.

4. WHO, 2006. Preventive Chemotherapy in Human Helminthiasis. Geneva: World Health Organization.

5. Stoltzfus RJ, Chway HM, Montresor A, Tielsch JM, Jape JK, Albonico M, Savioli L, 2004. Low dose daily iron supplementation improves iron status and appetite but not anemia, whereas quarterly anthelminthic treatment improves growth, appetite and anemia in Zanzibari preschool children. $J$ Nutr 134: $348-356$

6. Awasthi S, Peto R, Pande VK, Fletcher RH, Read S, Bundy DAP, 2008. Effects of deworming on malnourished preschool children in India: an open-labeled, cluster-randomized trial. PLoS Negl Trop Dis 2: e223.
7. Lustigman S, Prichard RK, Gazzinelli A, Grant WN, Boatin BA, McCarthy JS, Basáñez M-G, 2012. A research agenda for helminth diseases of humans: the problem of helminthiases. PLoS Negl Trop Dis 6: e1582.

8. UN-Habitat, 2003. The Challenge of Slums: Global Report on Human Settlements. London, UK: United Nations Human Settlements Programme.

9. Albonico M, Allen H, Chitsulo L, Engels D, Gabrielli AF, Savioli L, 2008. Controlling soil-transmitted helminthiasis in preschool-age children through preventive chemotherapy. PLoS Negl Trop Dis 2: e126.

10. Oria PA, Arunga G, Lebo E, Wong J, Emukule G, Muthoka P, Otieno N, Mutonga D, Breiman R, Katz M, 2013. Assessing parents' knowledge and attitudes towards seasonal influenza vaccination of children before and after a seasonal influenza vaccination effectiveness study in lowincome urban and rural Kenya, 2010-2011. BMC Public Health 13: 391.

11. Feikin D, Olack B, Bigogo G, 2011. The burden of common infectious disease syndromes at the clinic and household level from population-based surveillance in rural and urban Kenya. PLoS One 6: e16085.

12. Suchdev PS, Ruth LJ, Woodruff BA, Mbakaya C, Mandava U, Flores-Ayala R, Jefferds MED, Quick R, 2012. Selling Sprinkles micronutrient powder reduces anemia, iron deficiency, and vitamin A deficiency in young children in Western Kenya: a cluster-randomized controlled trial. Am J Clin Nutr 95: $1223-1230$.

13. WHO, 2011. Haemoglobin Concentrations for the Diagnosis of Anaemia and Assessment of Severity. Vitamin and Mineral Nutrition Information System. Geneva: World Health Organization.

14. Erhardt JG, Estes JE, Pfeiffer CM, Biesalski HK, Craft NE, 2004. Combined measurement of ferritin, soluble transferrin receptor, retinol binding protein, and C-reactive protein by an inexpensive, sensitive, and simple sandwich enzyme-linked immunosorbent assay technique. J Nutr 134: 3127-3132.

15. WHO, 2011. Serum Ferritin Concentrations for the Assessment of Iron Status and Iron Deficiency in Populations. Vitamin and Mineral Nutrition Information System. Geneva: World Health Organization.

16. Grant FKE, Suchdev PS, Flores-Ayala R, Cole CR, Ramakrishnari U, Ruth LJ, Martorell R, 2012. Correcting for inflammation changes estimates of iron deficiency among rural Kenyan preschool children. J Nutr 142: 105-111.

17. Thurnham DI, McCabe GP, Northrop-Clewes CA, Nestel P, 2003. Effects of subclinical infection on plasma retinol concentrations and assessment of prevalence of vitamin A deficiency: meta-analysis. Lancet 362: 2052-2058.

18. Zou G, 2004. A modified Poisson regression approach to prospective studies with binary data. Am J Epidemiol 159: 702-706.

19. WHO, 1998. Guidelines for the Evaluation of Soil-Transmitted Helminthiases and Schistosomiasis at Community Level. Geneva: World Health Organization.

20. Mwanthi MA, Kinoti MK, Wamae AW, Ndonga M, Migiro PS, 2008. Prevalence of intestinal worm infections among primary school children in Nairobi City, Kenya. East Afr J Public Health 5: 86-89.

21. Odiere MR, Opisa S, Odhiambo G, Jura WG, Ayisi JM, Karanja DM, Mwinzi PN, 2011. Geographical distribution of schistosomiasis and soil-transmitted helminths among school children in informal settlements in Kisumu City, Western Kenya. Parasitology 138: 1569-1577.

22. Hurst RJ, Else KJ, 2012. Retinoic acid signalling in gastrointestinal parasite infections: lessons from mouse models. Parasite Immunol 34: 351-359.

23. Atukorala TM, Lanerolle P, 1999. Soil-transmitted helminthic infection and its effect on nutritional status of adolescent schoolgirls of low socioeconomic status in Sri Lanka. $J$ Trop Pediatr 45: 18-22.

24. WHO, 2004. Strategy Development and Monitoring for Parasitic Diseases and Vector Control Team. How to Add Deworming to Vitamin A Distribution. Geneva: World Health Organization. 
25. Payne LG, Koski KG, Ortega-Barria E, Scott ME, 2007. Benefit of vitamin A supplementation on ascaris reinfection is less evident in stunted children. $J$ Nutr 137: 1455-1459.

26. Foote EM, Sullivan KM, Ruth LJ, Oremo J, Sadumah I, Williams TN, Suchdev PS, 2013. Determinants of anemia among preschool children in rural, western Kenya. Am J Trop Med Hyg 88: 757-764.

27. Kung'u JK, Goodman D, Haji HJ, Ramsan M, Wright VJ, Bickle QD, Tielsch JM, Raynes JG, Stoltzfus RJ, 2009. Early helminth infections are inversely related to anemia, malnutrition, and malaria and are not associated with inflammation in 6- to 23-month-old Zanzibari children. Am J Trop Med Hyg 81: 1062-1070.

28. WHO, 2012. Research Priorities for Helminth Infections: Technical Report of the TDR Disease Reference Group on Helminth Infections. Geneva: World Health Organization.
29. Allen JE, Maizels RM, 2011. Diversity and dialogue in immunity to helminths. Nat Rev Immunol 11: 375-388.

30. Taylor-Robinson DC, Maayan N, Soares-Weiser K, Donegan S, Garner P, 2012. Deworming drugs for soil-transmitted intestinal worms in children: effects on nutritional indicators, haemoglobin and school performance. Cochrane Database Syst Rev 7: CD000371.

31. Awasthi S, Peto R, Read S, Richards SM, Pande V, Bundy D; DEVTA (Deworming and Enhanced Vitamin A) Team, 2013. Population deworming every 6 months with albendazole in 1 million pre-school children in north India: DEVTA, a clusterrandomised trial. Lancet 381: 1478-1486.

32. Hawkes N, 2013. Deworming debunked. BMJ 346: e8558.

33. WHO, 2011. Helminth Control in School-Aged Children: A Guide for Managers of Control Programs. Geneva: World Health Organization. 